\title{
APPROXIMATION OF THE POLE FIGURES AND THE ORIENTATION OF DISTRIBUTION OF GRAINS IN POLYCRYSTALLINE SAMPLES BY MEANS OF CANONICAL NORMAL DISTRIBUTIONS
}

\author{
T. I. SAVYOLOVA \\ MIFI (Moscow Engineering Physical Institute), Kashirskoye shosse 31, \\ Moscow 115409, Russia
}

(Received 20 January, 1993)

The orientation distribution function (ODF) as determined from experimental pole figures (PF) in a polycrystalline sample by classical spherical harmonics analysis can have ghost effects and regions of negative values. The regions of negative values and the ghosts are a consequence of the loss of information on the "odd" part of ODF.

In the present paper the canonical normal distributions (CND) on the rotation group $S O(3)$ and on the sphere $S^{2}$ in $R^{3}$ used in texture analysis are discussed.

The examples of CND on $S O(3), S^{2}$ and their PF calculated for hexagonal lattice symmetry and for a rolling texture of beryllium are demonstrated.

KEY WORDS Canonical normal distribution (CND) on $S O(3)$ and $S^{2}$, ODF and PF approximation.

\section{INTRODUCTION}

The most widely applied method to solve the determination problem of the ODF from experimental pole figures is the series expansion formalism proposed by Bunge $(1969,1982)$. This method has ghost effects and regions of negative values (Matthies, 1979). The ghosts are a consequence of the inversion symmetry of experimental pole figures which leads to the loss of information on the "odd" part of ODF.

The ODF $f(g), g \in S O(3)$, is the solution of the equation (Matthies, 1979)

$$
\hat{P} f(g)=\left(8 \pi^{2}\right)^{-1} \int_{S O(3)}\left\{\delta\left(h_{i}-g y\right)+\delta\left(h_{i}+g y\right)\right\} f(g) d g=(4 \pi)^{-1} P_{h_{i}}(y),
$$

where $f(g)$ is the ODF, $P_{h_{i}}(y)$ is PF. The unit vector $h_{i}$ represents a crystal direction placed in the crystal coordinate system. The unit vector $y$ is a sample direction in the sample coordinate system.

Let ODF have the harmonic representation through the generalised spherical functions

$$
f(g)=\sum_{l=0}^{\infty} \sum_{m, n=-l}^{l} C_{m n}^{l} T_{m n}^{l}(g)
$$


where $C_{m n}^{l}$ are Fourier-type coefficients of $f(g)$. Coefficients $C_{m n}^{l}$ are determined from experimental pole figures $P_{h_{i}}(y)$ using the Eq. (1), where PF are represented as a series in spherical functions (Bunge, 1969).

If we get

$$
f(g)=f_{1}(g)+f_{2}(g),
$$

where $f_{1}(g)$ is the even part of ODF

$$
f_{1}(g)=\sum_{k=0}^{\infty} \sum_{m, n=-2 k}^{2 k} C_{m n}^{2 k} T_{m n}^{2 k}(g),
$$

$f_{2}(g)$ is the odd part of ODF

$$
f_{2}(g)=\sum_{k=0}^{\infty} \sum_{m, n=-(2 k+1)}^{2 k+1} C_{m n}^{2 k+1} T_{m n}^{2 k+1}(g),
$$

then we have $\hat{P} f_{2}(g)=0$ or $f_{2}(g) \in \operatorname{Ker} \hat{P}$. Thus the solution of Eq. (1) is non-unique.

There are different methods of calculation of ODF from PF (Bunge, 1969, 1982; Matthies, Wenk, Vinel, 1988; Savyolova, 1985).

We use normal distributions on the rotation group $S O(n)$ that have been defined by Parthasarathy (1964). These distributions are used for ODF approximations (Savyolova, 1984, 1989; Nikolayev et al., 1992). Definitions of normal distributions with different characteristics on the $S O(3)$ and $S^{2}$ can be found in Heyer (1977), Mardia (1972), Schaeben (1992).

In the present paper the canonical normal distributions are discussed (Savyolova, 1989). The canonical normal distributions are used for approximation of the pole figures and orientation distributions of grains in polycrystalline samples.

\section{DEFINITIONS OF THE CANONICAL NORMAL DISTRIBUTIONS ON $S O(3)$ AND $S^{2}$}

Let us consider the normal distributions on the rotation group $S O(3)$ as defined by Parthasarathy (1964), Savyolova (1984).

Definition 1 A distribution $\mu$ is said to be normal (Gaussian) if it admits representation of the type

$$
\int_{S O(3)} T_{g} d \mu(g)=\exp \left\{\sum_{i, j=1}^{3} \alpha_{i j} A^{i} A^{j}+\sum_{i=1}^{3} \alpha_{i} A^{i}\right\},
$$

where $T_{g}$ are the irreducible representations, $A^{i}$ are the infinitesimal operators of representations $T_{g},\left(\alpha_{i j}\right), i, j=1,2,3$, is a real positive definite or semidefinite matrix, and $\alpha_{i}, i=1,2,3$, are constants.

Let $g=\{\alpha, \beta, \gamma\}, 0 \leq \alpha, \gamma<2 \pi, 0 \leq \beta \leq \pi$ be three Eulerian angles that describe three rotations of Cartesian coordinates $\left(x_{1}, x_{2}, x_{3}\right)$ (right-handed) by rotation through angle $\alpha, 0 \leq \alpha<2 \pi$, about axis $O x_{3}$, then by rotation through angle $\beta, 0 \leq \beta \leq \pi$, about axis $O x_{1}$ and by third rotation through angle $\gamma, 0 \leq \gamma<$ $2 \pi$, about axis $O x_{3}$ (Vilenkin, 1965). 
Normal distribution $\mu(g), g \in S O(3)$ may be represented by the series (2), where $C_{m n}^{l}$ are the corresponding Fourier-type coefficients that can be calculated from expression (3) (Savyolova, 1984).

Definition 2 The normal distribution $\mu(g)$ on $S O(3)$ is said to be the canonical normal distribution, if we have $\alpha_{i j}=0, i \neq j, \alpha_{i}=0, i, j=1,2,3$, in the expression (3).

Then we have for CND

$$
B_{l}=\sum_{i=1}^{3} \alpha_{i i}\left(A_{l}^{i}\right)^{2}=\left(b_{i j}^{l}\right)
$$

where

$$
\begin{aligned}
b_{1+m, 1+m}^{l} & =b_{2 l+1-m, 2 l+1-m}^{l}=-\left[(2 m+1) l-m^{2}\right]\left(\alpha_{11}+\alpha_{22}\right) / 2-(l-m)^{2} \alpha_{33}, \\
b_{1+m, 3+m}^{l} & =b_{3+m, 1+m}^{l}=b_{2 l+1-m, 2 l-1-m}^{l}=b_{2 l-1-m, 2 l+1-m}^{l} \\
& =-[(m+1)(m+2)(2 l-m)(2 l-1-m)]^{1 / 2}\left(\alpha_{11}-\alpha_{22}\right) / 4,
\end{aligned}
$$

$m=0,1, \ldots, l$, and the rest $b_{i j}^{l}=0$.

By two rotations of the group $S O(3)$ we can get the CND from every normal distribution $\mu(g)$ (Nikolayev, Savyolova, 1986).

Using the definition $\exp B_{l}$ we can calculate the right-hand part of expression (3) for CND as the sum of series

$$
\exp B_{l}=E_{l}+B_{l}-\frac{1}{2 !} B_{l}^{2}+\ldots+\frac{1}{n !} B_{l}^{n}+\ldots
$$

where $E_{l}$ is the unit matrix of order $(2 l+1), l=1,2, \ldots$

If two matrices $A=\left(a_{i j}\right), B=\left(b_{i j}\right)$ of order $n, i, j=1,2, \ldots, n$, have $a_{i j}=b_{i j}=$ 0 with odd $(i+j)$, then for their product $C=A B=\left(c_{i j}\right)$ we have the same property $c_{i j}=0$ with odd $(i+j)$.

If two matrices $A=\left(a_{i j}\right), B=\left(b_{i j}\right), i, j=1,2, \ldots, n$, have elements with conditions $a_{i j}=a_{j i}=a_{n-i, n-j}=a_{n-j, n-i}, \quad b_{i j}=b_{j i}=b_{n-i, n-j}=b_{n-j, n-i}$, then their product $C=A B=\left(c_{i j}\right)$ has the same property $c_{i j}=c_{j i}=c_{n-i, n-j}=c_{n-j, n-i}$.

Thus we get these properties for matrices $\exp B_{l}, l=1,2, \ldots$, and consequently for coefficients of CND.

For the unitary matrix $B_{l}$ we obtain

$$
B_{l}=\left(D_{l}\right)^{-1} \Lambda_{l} D_{l},
$$

where $D_{l}$ is the orthogonal matrix, $\Lambda_{l}$ is the diagonal matrix. Thus we have

$$
\exp B_{l}=\left(D_{l}\right)^{-1} \exp \Lambda_{l}\left(D_{l}\right) \text {. }
$$

If $\alpha_{i}=0, \alpha_{i j}=0, i \neq j, \quad \alpha_{i i}=\varepsilon^{2}, j=1,2,3$, we obtain the central normal distribution on $S O(3)$ from formulas (3), (4)

$$
f(g)=\bar{f}_{1}(t)=\sum_{l=0}^{\infty}(2 l+1) \exp \left\{-l(l+1) \varepsilon^{2}\right\} \frac{\sin ((l+1 / 2) t)}{\sin (t / 2)},
$$

where $\cos (t / 2)=\cos (\beta / 2) \cos [(\alpha+\gamma) / 2]$.

In that case the CND's reduce to the Brownian motion distributions on $S O(3)$ (Roberts, Winch, 1984). 
The other normal distributions on $S O(3)$ we can get from formula (3) using the method of calculation (5).

Thus we can get coefficients $C_{m n}^{l}, l=0,1,2, \ldots$, only numerically except the central normal distributions (6).

When $l=0,1,2$, we can easily calculate the coefficients $C_{m n}^{l}$ from expression (3).

For the canonical normal distributions we have $C^{0}=1$. Then for $l=1$ we get from formulas (3), (4)

$$
\begin{aligned}
\sum_{m, n=-1}^{1} C_{m n}^{1} T_{m n}^{1}(g)= & 3 \exp \left\{-\left(\alpha_{11}+\alpha_{22}\right)\right\} \cos \beta+3\left[\exp \left\{-\left(\alpha_{11}+\alpha_{22}\right)\right\}\right. \\
& +\exp \left\{-\left(\alpha_{22}+\alpha_{33}\right\}\right] \cos [(\alpha+\gamma) / 2](1+\cos \beta) \\
& -3\left[\exp \left\{-\left(\alpha_{11}+\alpha_{33}\right)\right\}\right. \\
& \left.-\exp \left\{-\left(\alpha_{22}+\alpha_{33}\right)\right\}\right] \cos [(\alpha-\beta) / 2](1-\cos \beta)
\end{aligned}
$$

where $g=\{\alpha, \beta, \gamma\}, 0 \leq \alpha, \gamma<2 \pi, 0 \leq \beta \leq \pi$, are three Eulerian angles. When $l=2$ we have

$$
\begin{aligned}
C_{2,2}^{2}= & C_{-2,-2}^{2}=5 / 8 \omega\left[(2 \omega+\delta) \exp \left\{-\lambda_{1}\right\}+(2 \omega-\delta) \exp \left\{-\lambda_{2}\right\}\right. \\
& \left.+4 \omega \exp \left\{-\lambda_{5}\right\}\right], \\
C_{1,1}^{2}= & C_{-1,-1}^{2}=5 / 2\left(\exp \left\{-\lambda_{3}\right\}+\exp \left\{-\lambda_{4}\right\}\right), \\
C_{2,-2}^{2}= & C_{-2,2}^{2}=5 / 8 \omega\left[(2 \omega+\delta) \exp \left\{-\lambda_{1}\right\}+(2 \omega-\delta) \exp \left\{-\lambda_{2}\right\}\right. \\
& \left.-4 \omega \exp \left\{-\lambda_{5}\right\}\right], \\
C_{1,-1}^{2} C_{-1,1}^{2}= & 5 / 2\left(\exp \left\{-\lambda_{3}\right\}-\exp \left\{-\lambda_{4}\right\}\right), \\
C_{0,0}^{2}= & 5 / 4 \omega\left[(2 \omega-\delta) \exp \left\{-\lambda_{1}\right\}+(2 \omega+\delta) \exp \left\{-\lambda_{2}\right\}\right], \\
C_{0,2}^{2}= & C_{2,0}^{2}=C_{0,-2}^{2}=C_{-2,0}^{2}=5 \sqrt{6} / 8 \omega\left(\alpha_{11}-\alpha_{22}\right)\left(\exp \left\{-\lambda_{1}\right\}-\exp \left\{-\lambda_{2}\right\}\right),
\end{aligned}
$$

and the rest $C_{i j}^{2}=0$, where

$$
\begin{aligned}
\omega & =\left\{\sum_{i, j=1}^{3} \alpha_{i j}^{2}-\sum_{i, j=1, i \neq j}^{3} \alpha_{i i} \alpha_{j j}\right\}^{1 / 2}, \\
\delta & =\alpha_{11}+\alpha_{22}-2 \alpha_{33}, \\
\lambda_{1,2} & =2\left(\alpha_{11}+\alpha_{22}+\alpha_{33} \mp \omega\right), \\
\lambda_{3} & =4 \alpha_{11}+\alpha_{22}+\alpha_{33}, \\
\lambda_{4} & =4 \alpha_{22}+\alpha_{11}+\alpha_{33}, \\
\lambda_{5} & =4 \alpha_{33}+\alpha_{22}+\alpha_{11} .
\end{aligned}
$$

For $l=3,4$ we give the eigenvalues of matrices $B_{l}$ in Appendix. For $l \geq 5$ it is not difficult to calculate analytically the eignevalues of matrices $B_{l}$ by rotation method (Voevodin, 1977), but for determination of parameters $\alpha_{i i}, i=1,2,3$, it is enough to know coefficients $C_{m n}^{l}$ with $l=2,4$.

The normal distributions on sphere $S^{2}$ in $R^{3}$ can be obtained as "projections" from normal distributions on group $S O(3)$ (Nikolayev, Savyolova, 1986; Bucharova, Savyolova, 1993). We can get the functions on $S^{2}$ if we consider the functions on $S O(3)$ that are constant on right-hand contiquity classes for supgroup of rotation with respect to a certain axis. We take the angle of rotation $0 \leq \gamma<2 \pi$. 
Definition 3 We get a normal distribution $f(\xi), \xi=\{\alpha, \beta\}, 0 \leq \alpha<2 \pi$, $0 \leq \beta \leq \pi, \in S^{2}$, on the sphere $S^{2}$ in $R^{3}$ from normal distribution on the group $S O(3) \mu(g)$, where $\alpha_{3}=0, \alpha_{i j}=0$ if $i$ or $j=3, i, j=1,2,3$,

$$
\begin{aligned}
f(\xi) d \xi & =\left(8 \pi^{2}\right)^{-1} \int_{0}^{2 \pi}[f(g) \sin \beta d \alpha d \beta] d \gamma \\
& =(4 \pi)^{-1} \sum_{l=0}^{\infty} \sum_{m=-l}^{l} C_{m 0}^{l} T_{m 0}^{l}(g) \sin \beta d \alpha d \beta .
\end{aligned}
$$

If $\alpha_{i j}=0, i \neq j, \alpha_{i}=0, i, j=1,2, \alpha_{11}=\alpha_{22}=\varepsilon^{2}$ in expression (8), we have the central normal distributions on $S^{2}$

$$
f(\beta)=\sum_{l=0}^{\infty}(2 l+1) \exp \left\{-l(l+1) \varepsilon^{2}\right\} P_{l}(\cos \beta),
$$

where $P_{l}(\cos \beta)$ are Legendre polynomials. If $\alpha_{11} \neq \alpha_{22}, \alpha_{12}=\alpha_{21}=0$ in formula (8), we get the canonical normal distribution on the sphere $S^{2}$ in $R^{3}$.

THE CALCULATION OF THE CANONICAL NORMAL

\section{DISTRIBUTIONS ON $S O(3)$ AND ON $S^{2}$ AND THEIR POLE FIGURES}

Consider the calculation of pole figures for canonical normal distribution on $S O(3)$ for hexagonal lattice symmetry.

PF from CND in $S O(3)$ with $\alpha_{33}=0$ is the even part of CND on $S^{2}$ (Nikolayev, Savyolova, 1986). The proof of this property follows from definition of CND on $S^{2}(8)$, if we take $h_{i}=\{0,0\}$ coincident with axis $\mathrm{OZ}$ with angle of rotation $0 \leq \gamma<2 \pi$. Then for every pole figure without any crystal and sample symmetry we have the coefficients $C_{m n}^{l}$ from Eq. (1) for ODF (2) with even 1 only.

For hexagonal lattice symmetry the ODF's coefficients in formula (2) possess the following correlations

$$
\begin{aligned}
& C_{m n}^{l}=(-1)^{m+n} C_{-m,-n}^{l}, \\
& C_{m n}^{l}=(-1)^{n} C_{m,-n}^{l}, \\
& C_{m n}^{l}=0, \quad \text { if }\left[\frac{n}{6}\right]=0 \text {, where }\left[\frac{n}{6}\right] \text { is the whole part of } \frac{n}{6}, \\
& C_{m n}^{l}=0, \text { if } m \text { is an odd number, } m, n=-l,-l+1, \ldots, l .
\end{aligned}
$$

Using the properties of coefficients of the harmonic representation of CND, we get the correlations for coefficients $C_{m n}^{l}$ with $n=0$ that must be fulfilled for a polycrystalline sample of hexagonal lattice symmetry (Ratnikova, Savyolova, 1988). Thus if ODF is CND as defined from expression (3) with condition (4), we have the formula for PF $\{0001\}$

$$
P_{\{001\}}(y)=1+\sum_{l=1}^{\infty}\left(\frac{2}{2 l+1}\right)^{1 / 2}\left\{C_{00}^{2 l} P_{0}^{2 l}(\cos \chi)+2 \sum_{m=-2 l}^{2 l} C_{-m 0}^{2 l} P_{0}^{2 l}(\cos \chi) \cos m \eta\right\},
$$


where $y=\{\eta, \chi\}, 0 \leq \eta<2 \pi, 0 \leq \chi \leq \pi$, are the spherical coordinates of vector $y ; P_{m}^{2 l}(\cos \chi)$ are the spherical Legendre polynomials. If the coordinates of the centre of CND $f(g)$ are $g_{0}=\left\{\alpha_{0}, \beta_{0}, \gamma_{0}\right\}, 0 \leq \alpha_{0}, \gamma_{0}<2 \pi, 0 \leq \beta_{0} \leq \pi$, then instead of formula (2) we get

$$
f(g)=f\left(g_{0}^{-1} g\right)=\sum_{l=0}^{\infty} \sum_{m, n=-l}^{l} C_{m n}^{l} T_{m n}^{l}\left(g_{0}^{-1} g\right) .
$$

In this case we have the following formula for pole figure

$$
P_{h}(y)=\sum_{l=0}^{\infty} \frac{4}{4 l+1} \sum_{m, n=-2 l}^{2 l} C_{m n}^{2 l}(-1)^{m+n} Y_{m}^{2 l}(y)\left[Y_{n}^{2 l}\left(\Theta, \alpha_{0}-\varphi-\frac{\pi}{2}\right)\right]^{*},
$$

where $Y_{m}^{2 l}(y)$ are the spherical functions with $\cos \Theta=\cos \beta_{0} \cos \theta+$ $\sin \beta_{0} \sin \theta \sin \left(\gamma_{0}+\Phi\right), \quad \exp (i \varphi)=(\sin \Theta)^{-1}\left\{\sin \beta_{0} \cos \theta-\sin \beta_{0} \sin \left(\gamma_{0}+\Phi\right)-\right.$ $\left.i \sin \theta \cos \left(\Phi+\gamma_{0}\right)\right\}, 0 \leq \Theta \leq \pi, 0 \leq \varphi<2 \pi, h=\{\Phi, \theta\}, 0 \leq \Phi<2 \pi, 0 \leq \theta \leq \pi$, (Vilenkin, 1965).

For ODF approximation by CND we can find the coordinates of the centres of normal distributions for hexagonal lattice symmetry by the method described in Bucharova, Savyolova (1985); for cubic lattice symmetry see Nikolayev et al. (1992).

For ODF approximation by the canonical normal distributions the determination of the parameters of CND from pole figures is discussed in Savyolova (1989). The parameters of the CND $\left(\alpha_{11}, \alpha_{22}, \alpha_{33}\right)$ are determined from their coefficients $C_{m n}^{l}$ when $l=2(7)$.

Consider the following cases of resolving system (7) to determine parameters $\left(\alpha_{11}, \alpha_{22}, \alpha_{33}\right)$ for hexagonal lattice symmetry (Shalaev, Savyolova, 1989; Leyina, Savyolova, 1989).

1. Let $\alpha_{11}=\alpha_{22}=\alpha$. We have

$$
\alpha=(1 / 6) \ln \left(5 / C_{00}^{2}\right)
$$

We can not determine the parameter $\alpha_{33}$ from system (7). We can determine $\alpha_{33}$ from coefficient $C_{m n}^{l}$ from PF $\{10 \overline{10}\}$ with $l>2$, for example with $l=6$

$$
\alpha_{33}=(-1 / 36)\left[\ln \left(5 / C_{00}^{2}\right)+\ln \left(C_{66}^{6} / 13\right)\right] \text {. }
$$

It is easily to calculate the coefficients $C_{m n}^{l}$ of $\mathrm{CND}$ for $l>2$ in that case because the matrices $B_{l}(4)$ are diagonal.

2. Assume that we know $\alpha_{33}=\alpha$, where as $\alpha_{11} \neq \alpha_{22}$ are not known. We have two non-linear equations from (7) with coefficients $C_{00}^{2}, C_{-20}^{2}$ for determination of $\alpha_{11}, \alpha_{22}$. If $\left|\lambda_{1}\right|<1,\left|\lambda_{2}\right|<1$, we can calculate this system by Newton's method with the zero approximation

$$
\begin{aligned}
& \alpha_{11}^{0}=1 / 6-C_{00}^{2} / 30-C_{-20}^{2} / 5 \sqrt{6}, \\
& \alpha_{22}^{0}=1 / 6-C_{-20}^{2} / 5 \sqrt{6}-C_{00}^{2} / 30 .
\end{aligned}
$$

3. If $\alpha_{22}=\alpha$ is known (or $\alpha_{11}=\alpha$ is known), we have two equations with coefficients $C_{00}^{2}, C_{-20}^{2}$ for determination of parameters $\alpha_{11}, \alpha_{33}$. If $\left|\lambda_{1}\right|<1$, $\left|\lambda_{2}\right|<1$, we can use the zero approximation

$$
\begin{gathered}
\alpha_{11}^{0}=1 / 3-\alpha-C_{00}^{2} / 15, \\
\alpha_{33}^{0}=1 / 6-C_{00}^{2} / 15-2 \alpha-\left(C_{-20}^{2} / 5 \sqrt{6}\right)\left(1 / 3-2 \alpha-C_{00}^{2} / 15\right)^{-1}
\end{gathered}
$$

Then we can use Newton's method. 
Using the canonical normal distributions for ODF approximation we a priori suppose that ODF is the sum of several CND's. The unknown parameters in cases 2,3 can be calculated by selection method or from coefficients $C_{m n}^{l}$ with $l=4$.

\section{NUMERICAL RESULTS}

For calculation of CND on $S O(3)$ and $S^{2}$ with known parameters $\alpha_{i i}, i=1,2,3$, we used the QR algorithm (Voevodin, 1977) to find the eigenvalues and eigenvectors for matrices $B_{l}$ for $l \leq 16$. Using the formula (5) we get the coefficients $C_{m n}^{l}$ of CND from expression (3).

The canonical normal distributions on $S^{2}$ with parameters $\left(\alpha_{11}=1 / 4, \alpha_{22}=\right.$ 1/8), $\left(\alpha_{11}=1 / 4, \alpha_{22}=1 / 16\right)$ are calculated from formula $(8)$, where $T_{m 0}^{l}(g)=$ $P_{m 0}^{l}(\cos \beta) \exp \{-i m \alpha\}, 0 \leq \alpha<2 \pi, 0 \leq \beta \leq \pi$, in Figures $1 a ; 1 b$.

In Figures $2 a ; 2 b$ the CND on $S^{2}$ are shown with parameters $2 a(1 / 4,1 / 32) ; 2 b$ $(1 / 4,1 / 64)$.

The pole figures $\{0001\}$ for CND for hexagonal symmetry calculated from formula (10) with the same parameters as in Figures $1 a ; 1 b ; 2 a ; 2 b$ are shown in Figures $1 c ; 1 d ; 2 c ; 2 d$ respectively.

Figures $3 a ; 3 b ; 3 c ; 3 d$ demonstrate the pole figures $\{0001\}$ for CND on $S O(3)$ for hexagonal symmetry with parameters $a(1 / 4,1 / 16,1 / 4), b(1 / 4,1 / 16,1 / 8), c$ $(1 / 4,1 / 16,1 / 16), d(1 / 4,1 / 16,0)$.

For calculation of pole figures $\{0001\}$ for hexagonal symmetry we use formula (10) with coefficients $C_{m n}^{l}$ from (3), (4).

The pole figures $\{0001\}$ for hexagonal symmetry for ODF of the CND with parameters $4 a(1 ; 0 ; 0.3) ; 4 b(0 ; 1 ; 0.3)$ are demonstrated in Figures $4 a ; 4 b$ respectively.

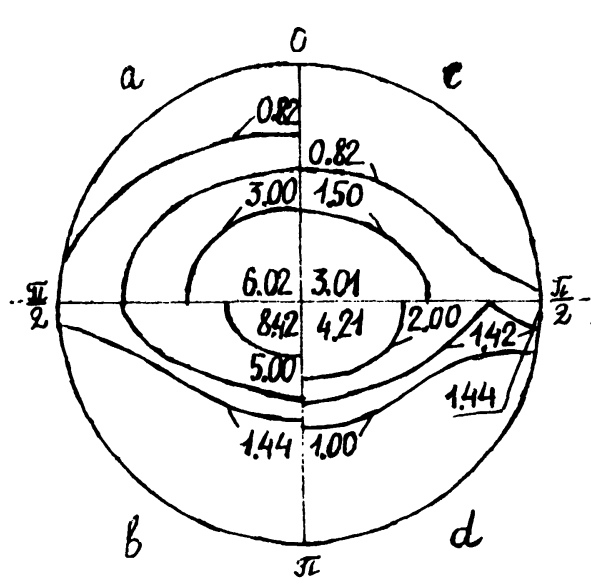

Figure 1 CND on $S^{2}$ with parameters $1 a$ $(1 / 4,1 / 8) ; 1 b(1 / 4,1 / 16)$; PF $\{0001\}$ for CND on $S^{2}$ with parameters $1 c$ the same as in Figure $1 a ; 1 d$ the same as in Figure $1 b$.

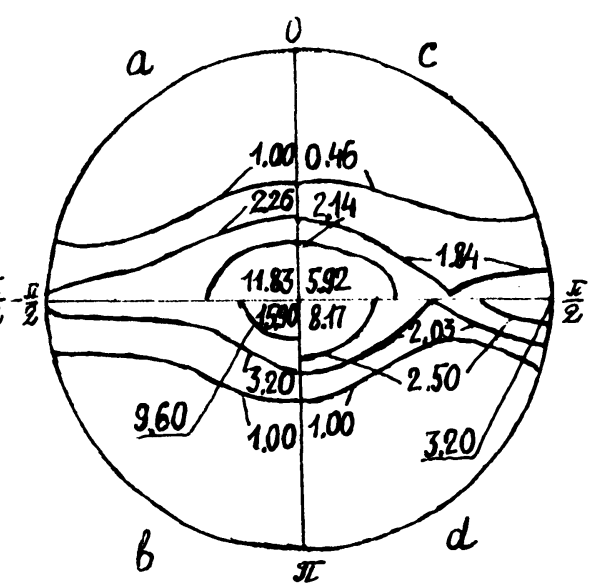

Figure 2 CND on $S^{2}$ with parameters $2 a$ $(1 / 4,1 / 32) ; 2 b(1 / 4,1 / 64)$; PF $\{0001\}$ for CND on $S^{2}$ with parameters $2 c$ the same as in Figure $2 a ; 2 d$ the same as in Figure $2 b$. 


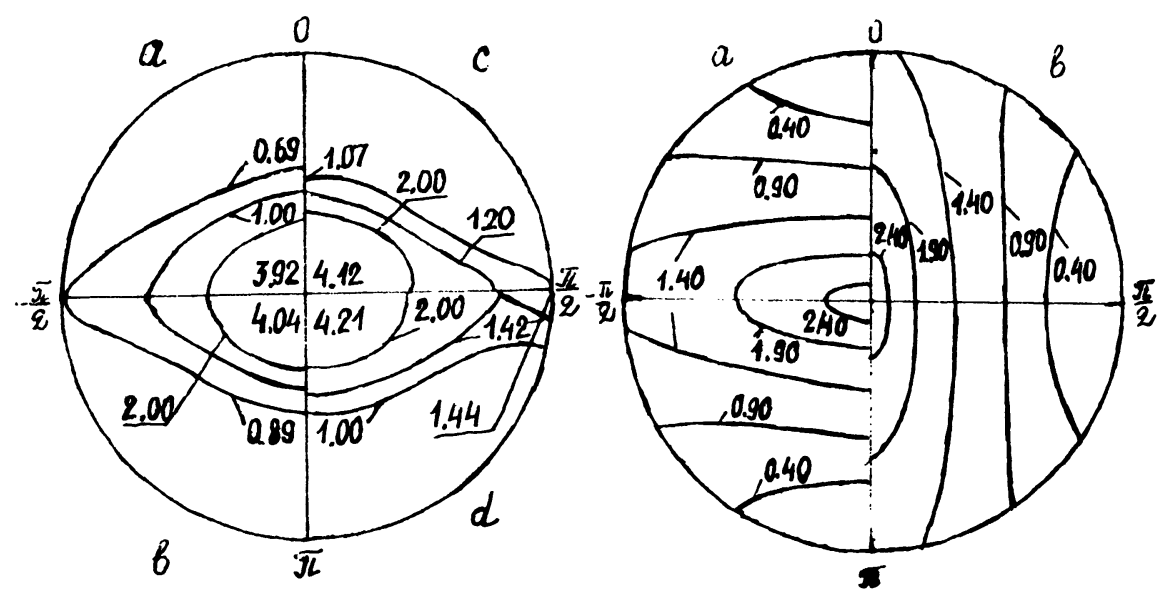

Figure 3 PF $\{0001\}$ for CND on $S O(3)$ with parameters $3 a(1 / 4,1 / 16,1 / 4) ; 3 b(1 / 4,1 / 16$, $1 / 8) ; 3 c(1 / 4,1 / 16,1 / 16) ; 3 d(1 / 4,1 / 16,0)$.

Figure 4 PF $\{0001\}$ for CND on $S O(3)$ with parameters $4 a(1,0,0.3), 4 b(0,1,0.3)$.

In Figure 5 the experimental pole figure $\{0001\}$ is shown for rolling texture of beryllium (continuous line) and the even part of CND on $S^{2}$ with parameters $(1 / 16,1 / 32)$ (dotted line).

The same as in Figure 5 is shown in Figure 6 with parameters $(1 / 4,1 / 16)$.

The error of the approximation of ODF by the CND was estimated by the

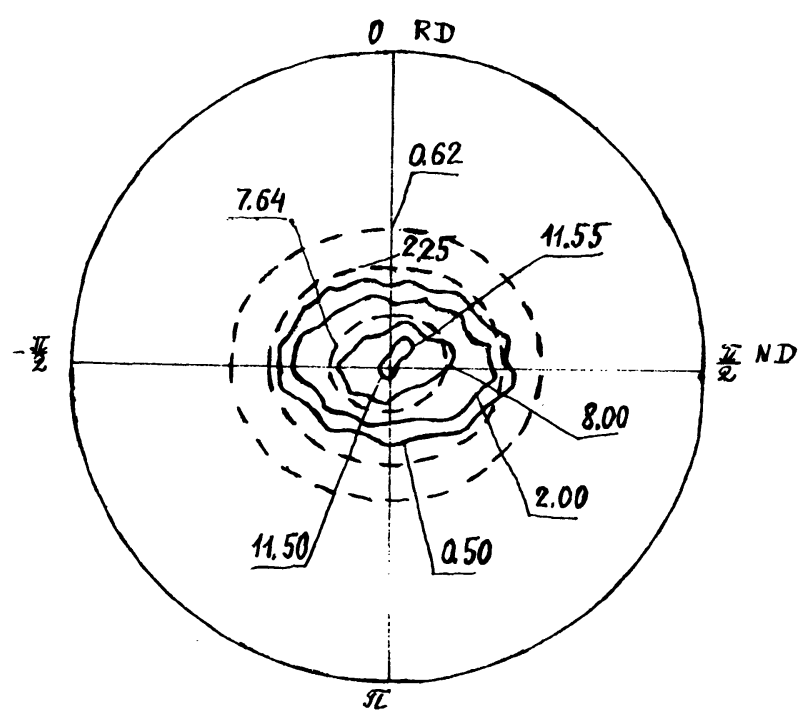

Figure 5 PF $\{0001\}$ for beryllium experimental data (continuous line); CND on $S^{2}$ with parameters $(1 / 16,1 / 32)$ (dotted line). 


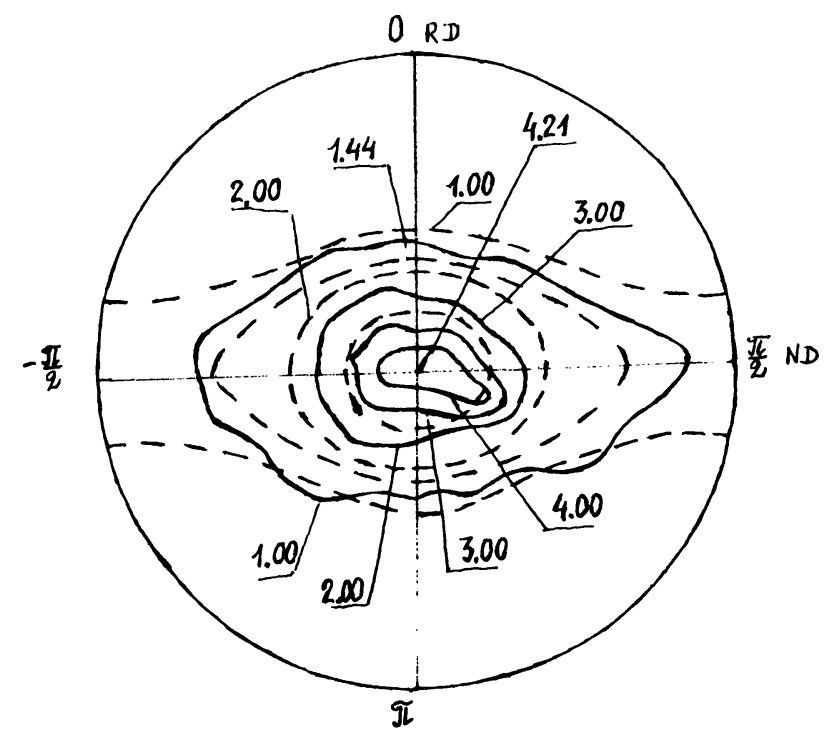

Figure 6 PF $\{0001\}$ for beryllium experimental data (continuous line); CND on $S^{2}$ with parameters $(1 / 4,1 / 16)$ (dotted line).

characteristic

$$
\delta^{2}=\frac{\int_{S^{2}}\left(P_{h}^{\exp }(y)-P_{h}^{\mathrm{CND}}(y)\right)^{2} d y}{\int_{S^{2}}\left(P_{h}^{\exp }(y)\right)^{2} d y}
$$

where $P_{h}^{\exp }(y)$ is experimental PF, $P_{h}^{\text {CND }}(y)$ is PF from CND. We get the following values of parameter $\delta: \delta=0.16$ in case of Figure $5, \delta=0.21$ for rolling texture of beryllium as shown in Figure 6 .

On the left-hand side of Figures 1,2 the CND's on $S^{2}$ are displayed with different parameters $\left(\alpha_{11}, \alpha_{22}\right)$ on the upper hemisphere $S^{2}$. They are close to zero on the lower hemisphere $S^{2}$. The even parts of CND's on $S^{2}$ with the same parameters $\left(\alpha_{11}, \alpha_{22}\right)$ are shown on the right-hand side of Figures 1,2. They coincide with PF $\{0001\}$ for hexagonal lattice symmetry (or also for a polycrystalline sample without any symmerty). We see that the maximum of pole figure is one half the maximum of CND on $S^{2}$.

Figure 4 shows that if instead of parameter $\alpha_{11}$ we put the parameter $\alpha_{22}$, and inversely, instead of parameter $\alpha_{22}$ we put $\alpha_{11}$, then the pole figure $\{0001\}$ rotates by an angle of $\eta=\frac{\pi}{2}$.

Figure 5,6 display examples of approximation of experimental PF $\{0001\}$ by the even part of CND on $S^{2}$. Using the coefficients $C_{m n}^{l}$ with $l=2$ of harmonic representation of experimental $P F\{0001\}$ as a series in spherical functions the parameters $\left(\alpha_{11}, \alpha_{22}\right)$ were found by the method described in case 2 with $\alpha_{33}=0$. 
By approximating the ODF by superposition of a number of CND we define the odd part of ODF by the odd part of superposition of CND. This ODF satisfies the positivity condition and is free of ghost effects, if the positions of components of texture are isolated.

\section{CONCLUSION}

In the present paper the canonical normal distributions on the group $S O(3)$ and on sphere $S^{2}$ are discussed. The central normal distributions on $S O(3)(6)$ and $S^{2}(9)$ have analytic formulas, other normal distributions on $S O(3)$ and $S^{2}$ can only be caluclated numerically. Numerical calculations of CND are demonstrated. The canonical normal distribution can be used for approximation of the orientation distribution function and pole figures. Numerical calculations of pole figures from CND for hexagonal lattice symmetry are demonstrated. When the experimental pole figures are approximated by $\mathrm{CND}$, the parameters of $\mathrm{CND}$ can be determined from coefficients $C_{m n}^{2}, m, n=-2,-1, \ldots, 2$. A drawback of this method is the non-linear problem of determination of parameters of CND.

The canonical normal distributions are not studies completely, but they are more flexible than the central normal distributions and they offer more possibilities for ODF and PF approximation.

\section{APPENDIX}

The eigenvalues of matrices $B_{t}=\left(b_{i j}^{l}\right)$ with elements (4) are (we drop the superscript $\left.l, b_{i j}^{l} \rightarrow b_{i j}\right)$ for $l=3$

$$
\begin{aligned}
\lambda_{1} & =b_{22}, \\
\lambda_{2,3} & =\frac{1}{2}\left\{\left(b_{22}+b_{44}\right) \pm\left[\left(b_{22}-b_{44}\right)^{2}+8 b_{24}^{2}\right]^{1 / 2}\right\}, \\
\lambda_{4,5} & =\frac{1}{2}\left\{\left(b_{11}+b_{33}-b_{35}\right) \pm\left[\left(b_{11}-b_{33}+b_{35}\right)^{2}+4 b_{13}^{2}\right]^{1 / 2}\right\} \\
\lambda_{6,7} & =\frac{1}{2}\left\{\left(b_{11}+b_{33}+b_{35}\right) \pm\left[\left(b_{11}-b_{33}-b_{35}\right)^{2}+4 b_{13}^{2}\right]^{1 / 2}\right\}
\end{aligned}
$$

for $l=4$ (Nikolayev D.I. calculated them)

$$
\begin{aligned}
\lambda_{1} & =\frac{1}{2}\left\{\left(b_{11}+\mu_{1}+\left[\left(b_{11}-\mu_{1}\right)^{2}+4 \beta_{1}^{2}\right]^{1 / 2}\right\},\right. \\
\lambda_{2,3} & =\frac{1}{2}\left\{\left(b_{22}+b_{44}+b_{46}\right) \pm\left[\left(b_{22}-b_{44}-b_{46}\right)^{2}+4 b_{24}^{2}\right]^{1 / 2}\right\}, \\
\lambda_{4,5} & =\frac{1}{2}\left\{\left(b_{22}+b_{44}-b_{46}\right) \pm\left[\left(b_{22}-b_{44}+b_{46}\right)^{2}+4 b_{24}^{2}\right]^{1 / 2}\right\}, \\
\lambda_{6,7} & =\frac{1}{2}\left\{\left(\delta+\mu_{2} \pm\left[\left(\delta-\mu_{2}\right)^{2}+4 \beta_{2}^{2}\right]^{1 / 2}\right\},\right. \\
\lambda_{8,9} & =\frac{1}{2}\left\{\left(b_{11}+b_{33} \pm\left[\left(b_{11}+b_{33}\right)^{2}+4 b_{13}^{2}\right]^{1 / 2}\right\},\right.
\end{aligned}
$$

where

$$
\begin{aligned}
\delta & =\frac{1}{2}\left\{\left(b_{11}+\mu_{1}-\left[\left(b_{11}-\mu_{1}\right)^{2}+4 \beta_{1}^{2}\right]^{1 / 2}\right\}\right. \\
\mu_{1,2} & =\frac{1}{2}\left\{b_{33}+b_{55} \pm\left[\left(b_{33}-b_{55}\right)^{2}+8 b_{35}^{2}\right]^{1 / 2}\right\} \\
\beta_{1,2} & =b_{13}^{2}\left\{1 \pm\left(b_{33}-b_{55}\right)\left[\left(b_{33}-b_{55}\right)^{2}+8 b_{35}^{2}\right]^{-1 / 2}\right\}
\end{aligned}
$$




\section{ACKNOWLEDGEMENT}

The author wishes to thank Dr. Kapcherin (Physical and Technical Institute, Kharkov, Ukraine) for the experimental data, the students of MIFI M. A. Shalaev, S. E. Leyina, T. A. Ratnicova for calculations of PF and CND.

The author would like to thank the reviewer for critical discussion of the manuscript.

\section{References}

Bucharova, T. I. and Savyolova, T. I. (1985). Approximation of the solution of one inverse diffraction problem. Journal of Numerical Mathematics and Mathematical Physics, 25, 617-622 (in Russian).

Bucharova, T. I. and Savyolova, T. I. (1993). Application of normal distributions on $S O(3)$ and $S^{n}$ for orientation distribution function approximation. Textures and Microstructures (in press).

Bunge, H. J. (1969). Mathematische Methoden der Texturanalyse. Academic-Verlag, Berlin.

Bunge, H. J. (1982). Texture Analysis in Materials Science. Butterworths, London.

Heyer, H. (1977). Probability Measures on Locally Compact Groups. Springer-Verlag, Berlin, Heidelberg, New-York.

Leyina S. E. and Savyolova, T. I. (1989). Reconstruction of Gaussian distributions of canonical type from PF. Mathematical modelling of problems of mechanics of continuous media. Energoatomizdat, Moscow 61-64 (in Russion).

Mardia, K. V. (1972). Statistics of Directional Data. Academic Press, London, New York.

Matthies, S. (1979). On the reproducibility of the orientation of function of texture samples from pole figures (Ghost Phenomena). Physica Status Solidi (b), 92, K135-K137.

Matthies, S., Wenk, H. R. and Vinel, G. W. (1988). Some basic concepts of texture analysis and comparison of three methods to calculate orientation distributions from pole figures. J. Appl. Cryst., 21, 285-304.

Nikolayev, D. I., Savyolova, T. I., Feldmann, K. (1992). Approximation of the orientation distributions of grains in polycristalline samples by means of Gaussians. Textures and Microstructures, 19, 9-27.

Nikolayev, D. I., Savyolova, T. I. (1986). Gaussian Distributions on $S O(3)$ and their Application in Texture Analysis. MIFI-Preprint 060-86, Moscow (in Russian).

Parthasarathy, K. P. (1964). The central limit theorem for the rotation group. Theory of Probabilities and its Application, 9, 273-282.

Ratnicova, T. A. and Savyolova, T. I. (1988). Gaussian Distributions on $S^{2}$ and Pole Figures. Preprint MIFI 090-88, Moscow (in Russian).

Roberts, P. H., Winch, D. E. (1984). On random rotation. Adv. Appl. Prob., 16, 638-665.

Savyolova, T. I. (1984). Distribution functions of grains with respect to orientation in polycrystals and their Gaussian approximations. Industrial Laboratory, 50, 468-474, translated from Zavodskaya Laboratoriya, 50, 48-52.

Savyolova, T. I. (1985). Preface to a new method of investigation of texture of polycrystalline samples. Sbornik perevodov statyej is zhurnala. Textures and Microstructures, Moscow, Metallurgiya (in Russian).

Savyolova, T. I. (1989). Computing polar figures and deriving orientation distributions from them for canonical Gaussian distributions. Industrial Laboratory, 55, 1045-1048, translated from Zavodskaya Laboratoriya, 55, 57-60.

Schaeben, H. (1992). Normal orientation distributions. Textures and Microstructures 19, 197-202.

Shalaev, M. A. and Savyolova, T. I. (1989). PF of Gaussian distributions of canonical type. Mathematical calculation and interpretation of results of physical experiments. Energoatomizdat, Moscow, 76-81 (in Russian).

Vilenkin, N. J. (1965). The special functions and theory of representations of groups. Science, Moscow (in Russian).

Voevodin, V. V. (1977). Numerical basis of linear algebra. Science, Moscow (in Russian). 